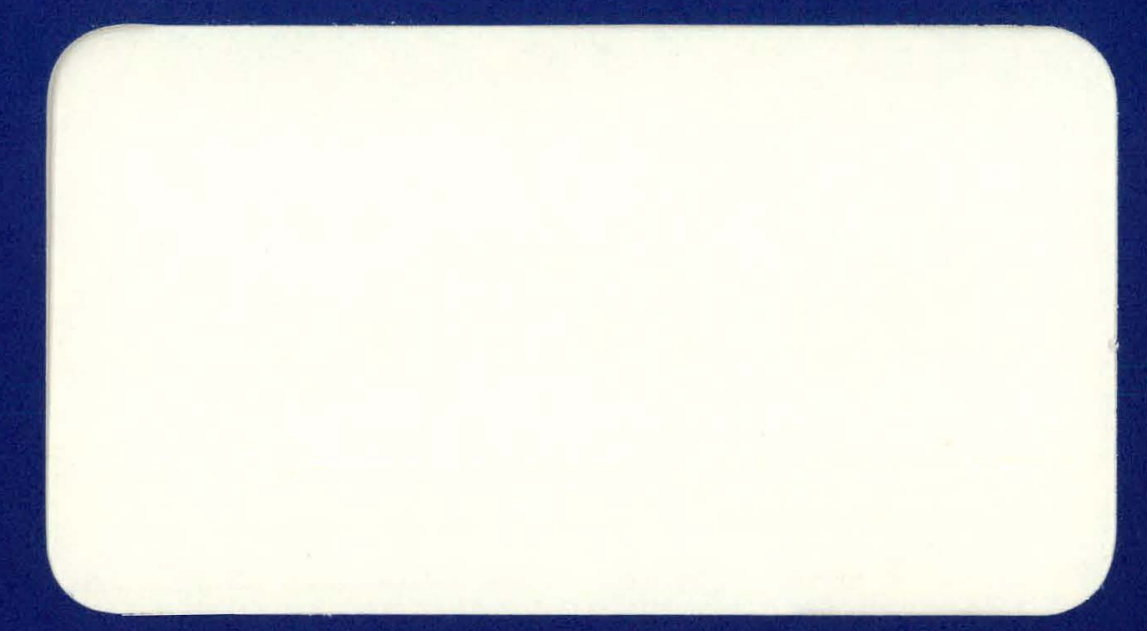

Westinghouse Nuclear Energy Systems

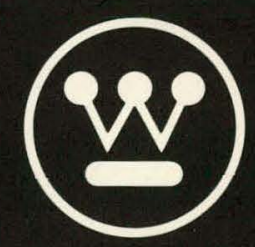




\section{DISCLAIMER}

This report was prepared as an account of work sponsored by an agency of the United States Government. Neither the United States Government nor any agency Thereof, nor any of their employees, makes any warranty, express or implied, or assumes any legal liability or responsibility for the accuracy, completeness, or usefulness of any information, apparatus, product, or process disclosed, or represents that its use would not infringe privately owned rights. Reference herein to any specific commercial product, process, or service by trade name, trademark, manufacturer, or otherwise does not necessarily constitute or imply its endorsement, recommendation, or favoring by the United States Government or any agency thereof. The views and opinions of authors expressed herein do not necessarily state or reflect those of the United States Government or any agency thereof. 


\section{DISCLAIMER}

Portions of this document may be illegible in electronic image products. Images are produced from the best available original document. 
This report was prepared as an account of work sponsored by the United States Government. Neither Commission, nor any their contractors, sub of their employees, nor any of makes any warranty legal liability or y, express or implied, or assumes any pleteness or usefulness of any for the accuracy, completeness or usefulness of any information, apparatus, would not infringe privately, or represents that its use i.

\title{
SAXTON PLUTONIUM PROJECT \\ QUARTERLY PROGRESS REPORT \\ FOR THE PERIOD ENDING \\ SEPTEMBER 30,1970
}

\section{ac -80}
Prepared for by the New York Operations Office
U. S. Atomic Energy Commission
Under AEC Contract AT (30-1)-3385

\author{
W. R. Smalley \\ Project Engineer
}

APPROVED :

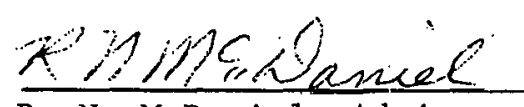

R. N. McDaniel, Advisory Engineer NFD Fuel Projects

\section{October 1970}

Printed in the United States of America Available from

National Technical Information Service Springfield, Virginia 22151

Price: Printed Copy $\$ 3.00$ M1crofiche $\$ 0.65$ 
NOTICE

This report was prepared as an account of work sponsored by the United States Government. Neither the United States nor the United States Atomic Energy Commission, nor any of their employees, nor any of their'contractors', subcontractors, or thèir employees, makes any warranty, express or implied, or assumes any legal liability or responsibility for the accuracy, completeness or usefulness of any information, apparatus, product or process disclosed, or represents that its use would not infringe privately-owned rights. 
EXTERNAL DISTRIBUTION

U. S. Atomic Energy Commission (45)

Brussels Office

23 Avenue des Arts

Brussels, Belgium

Attention: Senior AEC Representative

U. S. Atomic Energy Commission

New York Operations Office

376 Hudson Street

New York, New York 10014

Attention: Development Contracts Branch I (2)

Attention: Reports Library (1)

U. S. Atomic Energy Commission (1) Office of Foreign Activities, GM Washington, D. C. 20545

U. S. Atomic Energy Commisision (1) Assistant General Counsel for Patents Washington, D. C. 20545

U. S. Atomic Energy Commission (I)

R. D. T. Site Representative

P. O. Box 154

Madison, $\mathrm{Pa} ., 15663$

Attention: 'I' Iltis
U. S. Atomic Energy Commission (224)

Division of Technical Information

Extension

P. 0. Box 62

Oak Ridge, Tennessee 37830

U. S. Atomic Energy Commission (I) Brookhaven Office

Upton, L. I., New York 11973

Attention: Chief, Patent Group

U. S. Atomic Energy Commission (8) Division of Reactor Development \& Technology

Washington, D. C. 20545

Attention: Chief, Water Reactors Branch

Battelle Northwest Laboratory

P. O. Box 999

Richland, Washington 99352

Attention: Mr. M. D. Freshley 
TABLE OF CONTENTS

Section

Title

Page

1.0

INTRODUCTION AND SUMMARY . . . . . . . . . . . . 1-1

1.1 Scope . . . . . . . . . . . . . . . . . $1-1$

1.2 objectives . . . . . . . . . . . . . . 1-1

1.3 Project Administration . . . . . . . . . . . . 1-1

1.4 Summary of Progress During the Period . . . . . . . 1-1.

2.0 POST-IRRADIATION EXAMINATION OF CORE II FUEL . . . . . 2-1

3.0 SAXTON CORE III OPERATIONS . . . . . . . . . . . . 3-1

4.0 POST-IRRADIATION EXAMINATION OF CORE III FUEL . . . . 4-1

4.1 Examination of Rod RR . . . . . . . . . . . . 4-1

4.2 Mid-Life Sampling Plan . . . . . . . . . . 4-3 


\section{LIST OF ILLUSTRATIONS}

Figure

$2-1$

$2-2$

$2-3$

$3-1$

$4-1$

$4-2$
Title

Page

Calculated and Measured Uranium Isotopic Abundance versus Burnup in Saxton Core II Mixed-Oxide Fuel . . . . . . . . 2-4

Calculated and Measured Plutonium Isotopic Abundance versus Burnup in Saxton Core II Mixed-Oxide Fuel . . . 2-5

Calculated and Measured Pu/U Mass Ratio versus Burnup in Saxton Core II Mixed-0xide Fuel . . . . . . . . . . 2-6

Relative Assembly Power for Saxton Core III . . . . . . 3-2

Non-Uniform Surface near Center of Rod RR . . . . . . 4-2

Transition Area near Grid, 23.8 Inches from Bottom of

Rod RR . . . . . . . . . . . . . . . . . . 4-2 


\section{LIST OF TABLES}

Table

$2-1$

$2-2$

$2-3$

$2-4$

$2-5$

$2-6$

$3-1$

$4-1$

Title

Page

Summary of Saxton Core II Fuel Burnup . . . . . 2-2

Summary of Alpha Spectrometric Analyses for

$\mathrm{Pu}-236 / \mathrm{Pu}-239$ and $\mathrm{Pu}-238 / \mathrm{Pu}-239$ Atom Ratios in

Saxton Core II Fuel . . . . . . . . . . . 2-8

Summary of Radiochemical Analysis for $\mathrm{Np}-237$ in

Saxton Core II Fuel . . . . . . . . . . . 2-9

Summary of Analyses for Am-241 and Am-243 in

Saxton Core II Fuel . . . . . . . . . . 2-10

Summary of Alpha Spectrometric Analysis for $\mathrm{Cm}-242 / \mathrm{Pu}-239$ and $\mathrm{Cm}-244 / \mathrm{Pu}-239$ Atom Ratios in

Saxtion Core II Fuel . . . . . . . . . . . . 2-11

Comparison Between Measurement and Leopard-Hic

Calculation of $\mathrm{Np}, \mathrm{Pu}, \mathrm{Am}$ and $\mathrm{Cm}$ Isotopes in Saxton

Core II Mixed-Oxide Fuel. . . . . . . . . . 2-12

Summary of Saxton Core III Operating History-Third Quarter 1970.............. 3-3

Loose Lattice Fuel Rods to be Removed at Mid-Life for Destructive Examination . . . . . . . 4-3 
SECTION 1

INTRODUCTION AND SUMMARY

\subsection{SCOPE}

This quarterly report covers work completed on the Saxton Plutonium Project during the period July through September, 1970.

\subsection{OBJECTIVES}

The overall purpose of the Saxton Plutonium Project is to develop information concerning the utilization of plutonium-enriched fuel in pressurized water reactor systems. The program includes design, fabrication, operation, and post-irradiation examination of a partial plutonium core. The in-pile performance of the fuel will be evaluated and compared with analytical predictions.

\subsection{PROJECT ALMINISIRATION}

The quarterly report for the April-June 1970 period was written, reviewed, and released: The Saxton Core III schedule was revised to defer the mid-1ife shutdown to approximately 3500 effective full power hours (EPPH) and end-oflife to $\sim 6000$ to $7000 \mathrm{EFPH}$. Projected costs and distribution of costs for the Extended Burnup phase of the Saxton Plutonium Project were re-calculated to reflect the schedule changes. Post-irradiation examination of rod RR from the center $3 \times 3$ subassembly was added to the scope of the project. The Work Program was revised to reflect both of these changes and transmitted to the AEC.

\subsection{SUMMARY OF PROGRESS DURING THE PERIOD}

The physics section of the final report on Core II fuel evaluation was revised to reflect corrected values of $\mathrm{Pu}-239 / \mathrm{U}-238$ ratio. After the interim shutdown, 
the Saxton reactor operated at power for approximately 22 days during the quarter. As of September 30, the cumulative energy generated in Core III was 2133 megawatt-days, with a total of 184 load follow cycles. The peak burnups in the loose lattice region were:

(1) For peak power rod: 27,600 MWD/MTM

(2) For highest burnup rod: $37,100 \mathrm{MWD} / \mathrm{MTM}$

One of the four rods was removed from the center $3 \times 3$ subassembly during the interim shutdown. The rod was transferred to the WPIF Hot Laboratory, where it was visually examined and gamma scanned. The examination revealed no evidence of swelling, hydride blisters, cracks, or other defects. 
SECTION 2

EVALUATION OF CORE II FUEL

(R. J. Nodvik, W. R. Smalley, R. S. Miller)

Preparation and editing of drafts for the physics and materials sections of the final report on Cure II fuel evaluation were continued during the period. Significant revisions were made to the physics section reflecting the corrected values of $\mathrm{Pu}-239 / \mathrm{U}-238$ ratios which affect the comparison of burnups inferred by heavy element isotopic vs. Nd-148 and also the comparison of predicted and measured Pu-239 depletion.

As shown in Table 2-1, the comparisons of burnup values determined with two independent methods -- the Heavy Element method and the Nd-148 fission product method -- now show agreement to within two percent, (one percent reported previously[1]), thus verifying the internal consistency of the mass spectrometric data and the validity of the data-reduction methods.

Pellet burnups inferred from Cs-137 activity still show a 4 percent negative bias and indicate the possibility of slight Cs axial migration from the center to the ends of the fuel rods. The evaluation of Cs-137 data from radial microsamples continues to show considerable radial migration of cesium.

With the corrected values of $\mathrm{Pu}-239 / \mathrm{U}-238$, the comparisons between unit cell calculations and measurements for the main chain $U$ and $\mathrm{Pu}$ isotopes still show agreement to within about 3 percent. However, the calculations tend to underpredict the plutonium concentrations by $i 2$ percent. These results are summarized in Figures 2-1, 2-2, and 2-3.

[1] W. R. Smalley, "Saxton Plutonium Project Quarterly Progress Report for the Perlod Ending June 30, 1970", WCAP-3385-24, July 1970. 
TABLE 21

SUMMARY OF FUEL BURNUP

\begin{tabular}{|c|c|c|c|c|c|c|c|c|c|c|c|c|c|}
\hline \multirow[b]{3}{*}{ Assembly } & \multirow{3}{*}{$\begin{array}{l}\text { Fuel Rod } \\
\text { Lattice } \\
\text { Position }\end{array}$} & \multirow{3}{*}{$\begin{array}{l}\text { Fual } \\
\text { Rod } \\
\mathrm{Na}\end{array}$} & \multirow{3}{*}{$\begin{array}{l}\text { Fuel }^{(a)} \\
\text { Type }\end{array}$} & \multirow{3}{*}{$\begin{array}{l}\text { Neutron (b) } \\
\text { Spectrum }\end{array}$} & \multirow{3}{*}{$\begin{array}{l}\text { Axial } \\
\text { Sample } \\
\text { Zone }\end{array}$} & \multirow{3}{*}{$\begin{array}{l}\text { Axial|c| } \\
\text { Location } \\
\text { (Inches) }\end{array}$} & \multirow{3}{*}{$\begin{array}{l}\text { Sample }{ }^{(d)} \\
\text { I.D. }\end{array}$} & \multirow{2}{*}{\multicolumn{2}{|c|}{ Fuel Burnup (MWD'MTM) $(\mathrm{e})$}} & \multirow[b]{3}{*}{ Cs Muthod } & \multicolumn{3}{|c|}{$\begin{array}{l}\text { Ratio of Burnup } \\
\text { by Two Methods }\end{array}$} \\
\hline & & & & & & & & & & & \multirow{2}{*}{$\frac{H E}{N d}$} & \multirow{2}{*}{$\frac{\mathrm{Cs}_{s}}{\mathrm{Nd}}$} & \multirow{2}{*}{$\frac{C_{s}}{H E}$} \\
\hline & & & & & & & & Nd $M: 1 h w d$ & HE Method & & & & \\
\hline \multirow[t]{8}{*}{ C3 } & \multirow[t]{8}{*}{ E4 } & \multirow[t]{8}{*}{ RI } & \multirow[t]{8}{*}{$\mathbf{P}$} & \multirow[t]{8}{*}{ Asymp. } & 1 & 35.6 & RI. 18 & $7.240 \pm 230$ & $7,360 \pm 400$ & $6,910=610$ & 1.016 & 0.954 & 0.939 \\
\hline & & & & & 2 & 29.3 & RI.19 & $14.550 \pm 460$ & $14,920 \pm 760$ & $13,960=1,230$ & 1.025 & 0.959 & 0.936 \\
\hline & & & & & 3 & 24.3 & RI. 20 & $16,760 \pm 530$ & $\cdot 17,370 \pm 880$ & $16,380 \fallingdotseq 1,440$ & 1.036 & 0.977 & 0.943 \\
\hline & & & & & 4 & 21.3 & RI.21 & $19.670 \pm 620$ & $20.200 \pm 1.020$ & $-\infty----\infty \cdots$ & 1.027 & $\ldots$ & $-\ldots-$ \\
\hline & & & & & 6 & 19.3 & RI.3 & $20.450 \pm 640$ & $21,060 \pm 1,060$ & $19.480 \pm 1.710$ & 1.030 & 0.952 & 0.925 \\
\hline & & & & & 8 & 17.3 & RI.22 & $20,560 \pm 640$ & $21.170 \pm 1.070$ & $18,960 \pm 1,670$ & 1.030 & 0.922 & 0.896 \\
\hline & & & & & 10 & 9.3 & RI.24 & $17,600 \pm 550$ & $13.240 \pm 920$ & $16,680 \pm 1,470$ & 1.036 & 0.948 & 0.914 \\
\hline & & & & & 11 & 2.4 & R1.25 & $9,350 \div 300$ & $9.690 \pm 510$ & $9,210 \pm 820$ & 1.036 & 0.985 & 0.950 \\
\hline \multirow[t]{2}{*}{ C3 } & \multirow[t]{2}{*}{ E5 } & \multirow[t]{2}{*}{$J F$} & \multirow[t]{2}{*}{$\mathbf{P}$} & \multirow[t]{2}{*}{ Asymp. } & 7 & 18.3 & $J F \cdot I$ & $20.110 \pm 630$ & $20,640 \pm 1,040$ & .--ー-ー-ー--- & 1.026 & --- & $-\ldots$ \\
\hline & & & & & 10 & 9.3 & $J F-27$ & $17,620 \pm 550$ & $18,040 \pm 910$ & ----------- & 1.024 & ---- & --- \\
\hline \multirow[t]{3}{*}{ C3 } & \multirow[t]{3}{*}{05} & \multirow[t]{3}{*}{$M^{2}$} & \multirow[t]{3}{*}{$\mathbf{P}$} & \multirow[t]{3}{*}{ Asymp. } & 2 & 29.3 & MY-16 & $14,600 \pm 460$ & $14,960 \pm 760$ & $\ldots$ & 1.025 & ---- & ---- \\
\hline & & & & & 6 & 19.3 & $M Y \cdot 2$ & $20,230 \pm 630$ & $20,740 \pm 1,050$ & -- - - - - - - & 1.025 & ---- & $\ldots$ \\
\hline & & & & & 10 & 9.3 & MY.17 & $16,700 \pm 530$ & $17.880 \pm 910$ & ------- & 1.071 & ---- & ---- \\
\hline C3 & D1 & TE & $\mathbf{p}$ & FW/WS & 2 & 29.3 & TE.14 & $20,070 \pm 640$ & $20,770 \pm 1,050$ & $-\infty+\cdots--$ & 1.035 & .... & $\ldots$ \\
\hline & & & & & 6 & 19.3 & TE.4 & $27,730 \pm 870$ & $28,660 \pm 1,450$ & - & 1.034 & $--\cdots$ & $--\infty$ \\
\hline & & & & & 10 & 9.3 & TE-15 & $24.460 \pm 770$ & $24,970 \pm 1,260$ & ---+----- & 1.021 & ---- & ---- \\
\hline C3 & A1 & $\mathrm{OE}$ & $P$ & WS/ws & 6 & 19.3 & 7405 & $27,550 \pm 860$ & $28,810 \pm 1,450$ & ---------- & 1.046 & --- & ---- \\
\hline & & & & & & & 640 & $27,720 \pm 870$ & $26,250 \pm 1.410$ & - - - & 0.947 & ---- & --- \\
\hline E2 & A1 & LF. & $\mathbf{P}$ & WS & 6 & 19.3 & LA.11 & $24.070 \pm 760$ & $24.710 \pm 1.250$ & -----_------- & 1.026 & $\therefore---$ & ….-- \\
\hline D3 & $A 1$ & $\mathrm{TI}$ & $\mathbf{P}$ & WS/WS & 6 & 19.3 & $T 1.8$ & $25,980 \pm 810$ & $26,560 \pm 1,340$ & - & 1.022 & $\ldots-\cdots$ & $\cdots \cdot \cdot--$ \\
\hline D3 & 46 & $T T:$ & $\mathbf{P}$ & $\mathrm{FW}$ & 6 & 19.3 & TT.10 & $27,180 \pm 860$ & $28,170 \pm 1,430$ & ...-_----- & 1.036 & $\ldots$ & --_- \\
\hline D3 & 01 & $T P$ & $P$ & FW/WS & 6 & 19.3 & TP.9 & $26,990 \pm 850$ & $27,590 \pm 1,390$ & $-\cdots+---$ & 1.022 & $-\cdots$ & ---- \\
\hline D3 & D9 & $\mathrm{Cr}$ & $\mathbf{P}$ & FW/WS & 6 & 19.0 & 7403 & $27,960 \pm 880$ & $28,150 \pm 1.420$ & 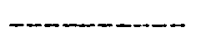 & 1.007 & $\therefore$ & $-\cdots$ \\
\hline & & & & & & & 680 & $28,180 \pm 880$ & $27,250 \div 1,460$ & $-\cdots$ & 0.967 & $-\cdots$ & $---\cdot$ \\
\hline
\end{tabular}


TABLE $2 \cdot 1$ (CONT)

SUMMIARY OF FUEL BURNUP

\begin{tabular}{|c|c|c|c|c|c|c|c|c|c|c|c|c|c|}
\hline \multirow[b]{2}{*}{ Assembly } & \multirow{2}{*}{$\begin{array}{l}\text { Fuel Rod } \\
\text { Lattice } \\
\text { Position }\end{array}$} & \multirow{2}{*}{$\begin{array}{l}\text { Fuel } \\
\text { Rod } \\
\text { No. }\end{array}$} & \multirow[b]{2}{*}{$\begin{array}{l}\text { Fuel (a) } \\
\text { Type }\end{array}$} & \multirow[b]{2}{*}{$\begin{array}{l}\text { Neutro, }(b) \\
\text { SpectrLm }\end{array}$} & \multirow{2}{*}{$\begin{array}{l}\text { Axial } \\
\text { Sample } \\
\text { Zone }\end{array}$} & \multirow{2}{*}{$\begin{array}{l}\text { Axial }(c) \\
\text { Lociation } \\
\text { linches) }\end{array}$} & \multirow[b]{2}{*}{$\begin{array}{l}\text { Sample }{ }^{(d)} \\
\text { I.D. }\end{array}$} & \multicolumn{3}{|c|}{ Fiet Burnup) (MWSiMTM) } & \multicolumn{3}{|c|}{$\begin{array}{l}\text { Rutios of Burnup } \\
\text { by Two Methods }\end{array}$} \\
\hline & & & & & & & & Nu Method & HE Method & Cs Method & $\frac{\mathrm{HE}}{\mathrm{Nd}}$ & $\frac{C_{s}}{N d}$ & $\frac{\mathrm{Cs}}{\mathrm{HE}}$ \\
\hline D4 & D5 & \$1 & v & Asymp & $\begin{array}{r}1 \\
2 \\
6 \\
10 \\
11\end{array}$ & $\begin{array}{r}35.5 \\
29.3 \\
19.3 \\
9.3 \\
2.4\end{array}$ & $\begin{array}{l}51.28 \\
51.29 \\
51.7 \\
51.30 \\
51.31\end{array}$ & $\begin{aligned} 7,520 & \pm 240 \\
14,820 & \pm 470 \\
21,150 & \pm 660 \\
18,710 & \pm 590 \\
9,840 & \pm 310\end{aligned}$ & $\begin{aligned} 7.450 & \pm 400 \\
15.060 & : 770 \\
21,860 & \pm 1,100 \\
19.120 & \pm 970 . \\
10,160 & \pm 530\end{aligned}$ & - & $\begin{array}{l}0.991 \\
1.016 \\
1.034 \\
1.022 \\
1.032\end{array}$ & $\begin{array}{l}---- \\
\cdots \cdots- \\
\cdots-.- \\
-\cdots- \\
-\cdots\end{array}$ & $\begin{array}{l}\cdots-\cdots \\
\cdots-- \\
\cdots-- \\
\cdots--\end{array}$ \\
\hline D4 & E5 & PO & $v$ & Asymp. & $\begin{array}{r}2 \\
6 \\
8 \\
10\end{array}$ & $\begin{array}{r}29.3 \\
19.3 \\
17.3 \\
9.3\end{array}$ & $\begin{array}{l}\text { PO.32 } \\
\text { PO. } 6 \\
\text { PO. } 33 \\
\text { PO.34 }\end{array}$ & $\begin{array}{l}15,320 \pm 480 \\
: 1, ; 80 \pm 650 \\
21,080 \pm 660 \\
18,200 \pm 570\end{array}$ & $\begin{array}{l}15,840 \pm 810 \\
21.120 \pm 1,070 \\
21,630 \pm 1,090 \\
18,860 \pm 950\end{array}$ & - & $\begin{array}{l}1.034 \\
1.016 \\
1.026 \\
1.036\end{array}$ & -.... & 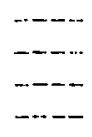 \\
\hline D4 & A1 & $K 4$ & $v$ & WSNWS & 6 & 19.3 & $K 4.5$ & $27.160 \pm 850$ & $28.080 \pm 1.420$ & - & 1.034 & ---- & $\cdots$ \\
\hline D4 & $A 2$ & N3 & $v$ & WS & 6 & 29.3 & N3.13 & $25.710 \pm 810$ & $26.540 \pm 1,340$ & -n- & 1.032 & $-\cdots--$ & --- \\
\hline D4 & A4 & NI & $V$ & FWNS & 6 & 19.3 & $N 1 \cdot 12$ & $28,900 \pm 910$ & $27.620 \pm 1.400$ & $-\cdots---\cdots----$ & 0.956 & $\cdots-\cdots$ & $\cdots-\cdots$ \\
\hline $\begin{array}{l}\text { D3 } \\
\text { D3 } \\
\text { E3 }\end{array}$ & $\begin{array}{l}2 \times 2 \\
2 \times 2 \\
E 1\end{array}$ & $\begin{array}{l}\text { A } \\
B \\
D\end{array}$ & $\begin{array}{l}P \\
P \\
P\end{array}$ & $\begin{array}{l}\text { Pert } \\
\text { Pert } \\
\text { FWWS }\end{array}$ & $\begin{array}{l}6 \\
6 \\
6\end{array}$ & $\begin{array}{l}19.5 \\
19.5 \\
19.5\end{array}$ & $\begin{array}{l}7401 \\
7402 \\
7404\end{array}$ & $\begin{array}{l}21,170 \pm 670 \\
19,700 \pm 620 \\
13,890 \pm 440\end{array}$ & $\begin{aligned} 20,510 & \pm 1,040 \\
19,790 & \pm 1,000 \\
13,180 & \pm 680\end{aligned}$ & - & $\begin{array}{l}0.969 \\
1.004 \\
0.949\end{array}$ & $\begin{array}{l}-\cdots- \\
---- \\
----\end{array}$ & - \\
\hline & & $\begin{array}{l}\text { Avera } \\
\text { Averia } \\
\text { Avero }\end{array}$ & $\begin{array}{l}\text { ge Ratio } \\
\text { fe Ratio } \\
\text { ge Ratio }\end{array}$ & $\begin{array}{l}\text { or Pellet Sim } \\
\text { or Viplac. Sam } \\
\text { or all Sample }\end{array}$ & & & & & & & $\begin{array}{l}1.018 \\
1.019 \\
1.018\end{array}$ & $\begin{array}{l}0.957 \\
-\cdots--\end{array}$ & 0.929 \\
\hline
\end{tabular}

Notes: (a) Pulke:ized and vibratory compacted ftel indicated by $P$ and $V$, respectively.

(b) Asynip indicates that the fuel rod was locited in the asymptotic neutron spectrum in the inner region of the fuel assembly: FW and WS indicate non-isymptotic neutron spectra adjacent to a flux.wire thimble and/or a water slot; and Pert indicates per:urbed recutron spectrum in $2 \times 2$ Overpower Test subassembly.

(c) Distance from bottom luel rod to mit.point of one.inch sample segment. Subtract 0.75 inches to obtain distance from bot:cm of tuel columr.

(d) All measured data used in burnup determination obtained from WARD, except mass spectrometric data from cross check analyses by GE.Vallecitos on samples QE. 640 and $\mathrm{CH} \cdot 680$.

(e) Uni:s are megawatt-days per $10^{6}$ grams initial loading of $U$ and Pu metal. Values of MeV/fission used to convert fissions to energy units include kinetic energy of fission fragnents and neutrons, energy of prompt and delayed gamma and beta decay. and energy of radiative capture in fued, cladding, and moderator. 


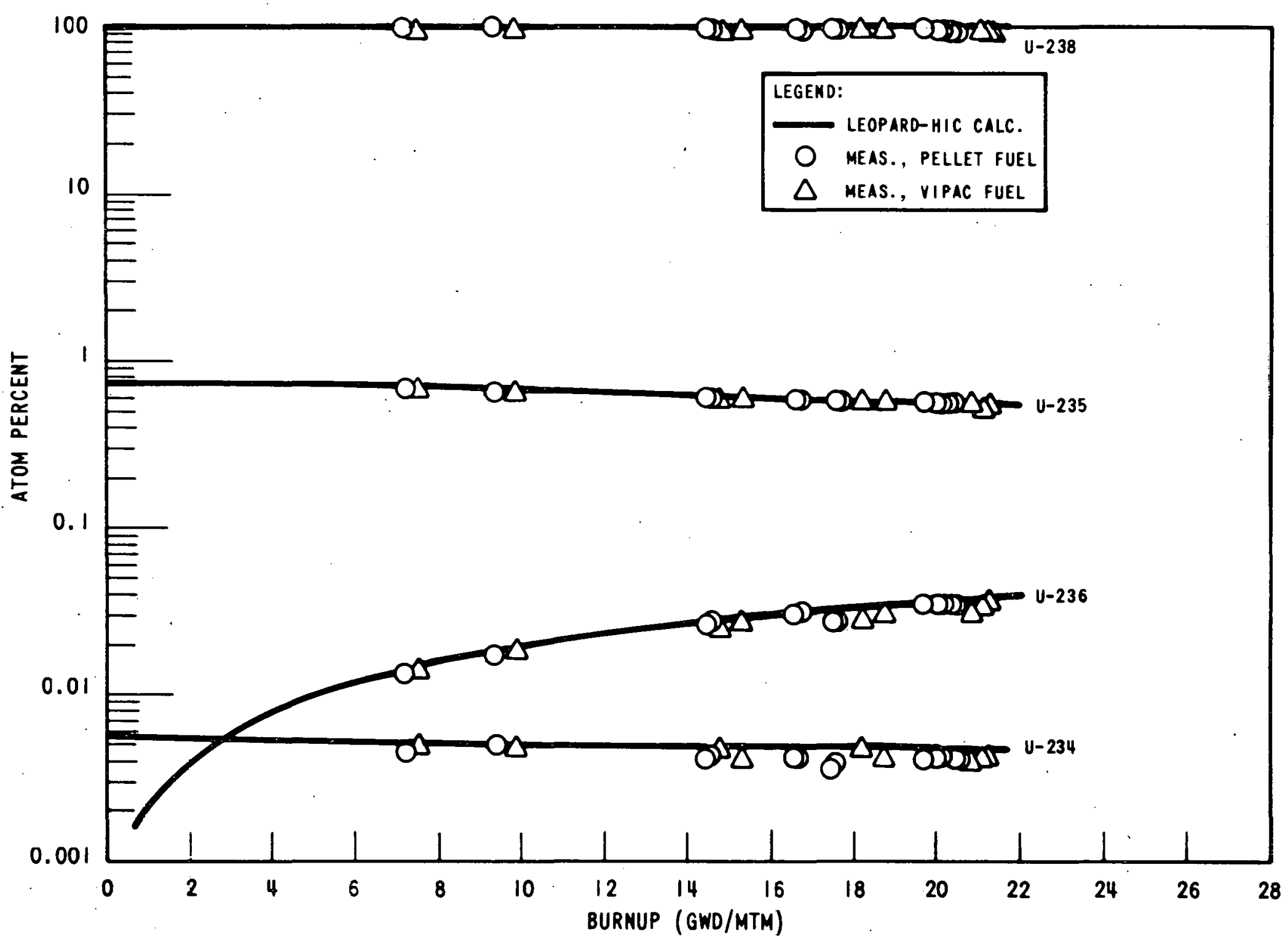

Figure 2-1. Calculated and Measured Uranium I sotopic Abundance versus Burnup in Saxton Core II Mixed-Oxide Fuel 


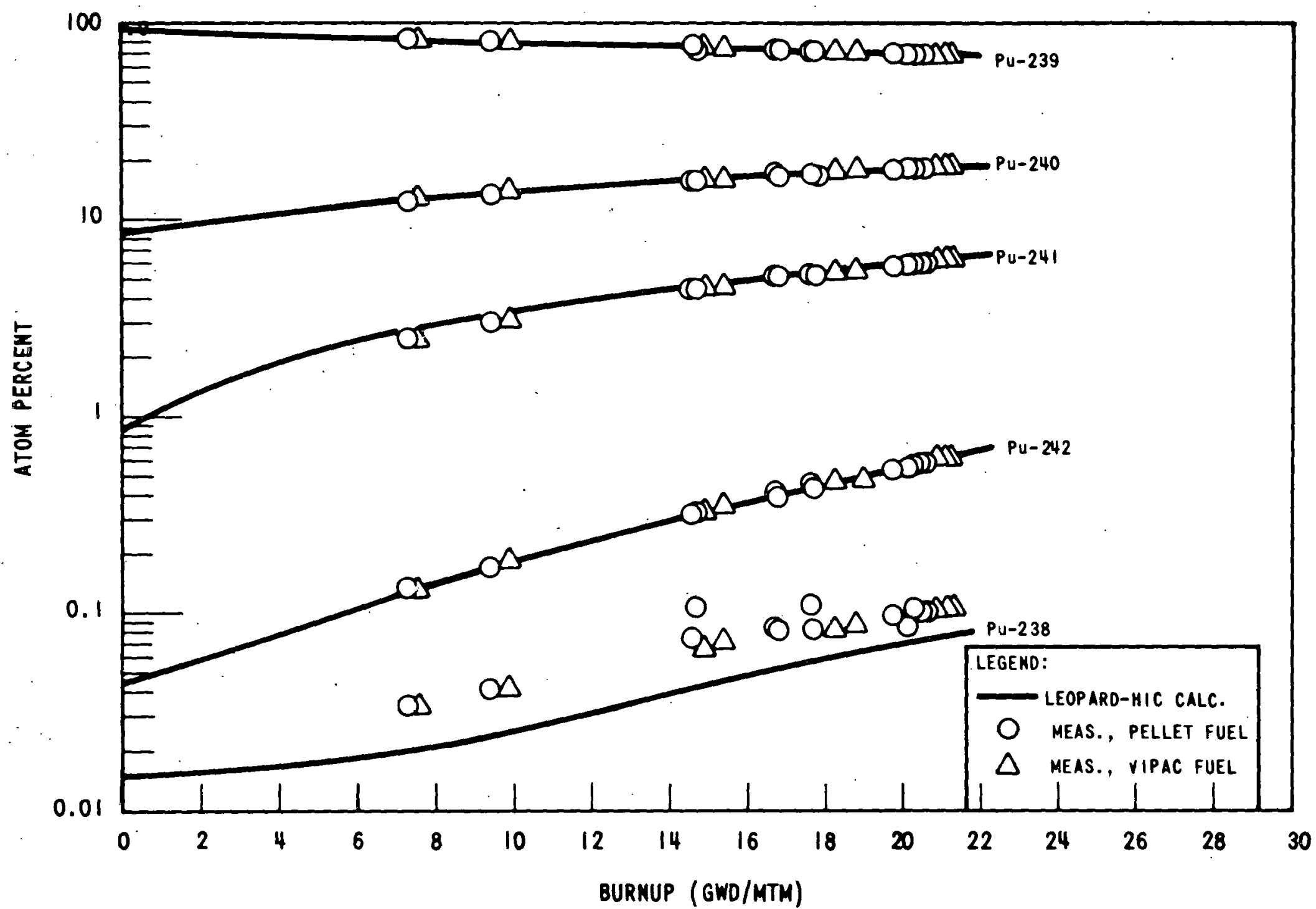

Figure 2-2. Calculated and Measured Plutonium Isotopic Abundance versus Burrup in Saxton Core II Mixed-Oxide Fuel 


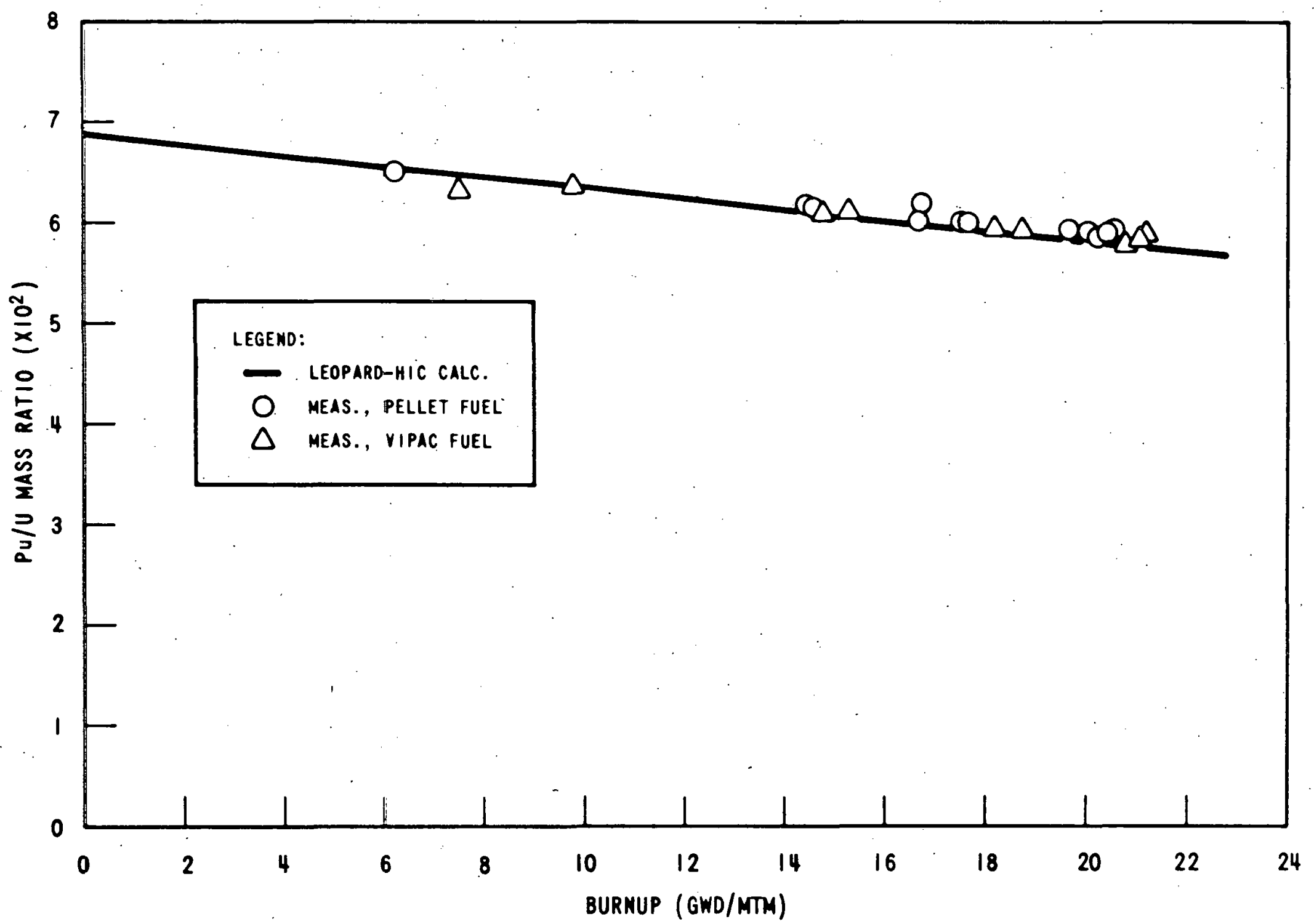

Figure 2-3. Calculated and Measured Pu/U Mass Ratio versus Burnup in Saxton Core II Mixed Oxide Fuel 
The results of measurements for special isotopes (1.e. Pu-236, Pu-238, $\mathrm{Np}-237, \mathrm{Am}-241, \mathrm{Am}-243, \mathrm{Cm}-242$, and $\mathrm{Cm}-244$ ) are presented in Tables 2-2 through 2-5. The alpha spectrum resolution obtained for the Am-243 samples was not sufficient to detect the Am-243 peak in the presence of the relatively large Am-241 peak; therefore, only maximum values of Am-243 can be reported. As reported previously ${ }^{(1)}$, the measured values of Pu-236 and $\mathrm{Am}-241$ are in good agreement with those predicted by unit cell calculations, while the Np-237 and Pu-238 are about 30 percent above the calculated values. The measurements of $\mathrm{Cm}-242 / \mathrm{Pu}-239$ and $\mathrm{Cm}-244 / \mathrm{Pu}-239$, determined by alpha spectrometric analysis of six samples from two asymptotic rods, indicate that unit cell calculations underpredict the $\mathrm{Cm}-242$ and $\mathrm{Cm}-244$ isotopes by approximately 20 and 40 percent, respectively. Table 2-6 summarizes the differences between measurements and unit cell predictions for each of the special isotopes.

(1) Ibid. 
TABLE 2-2

SUMMARY OF ALPHA SPECTROMETRIC ANALYSES FO'R Pu-236/Pu-239

AND PU-238/Pu-239 ATOM RATIOS IN SAXTON CORE II FUEL

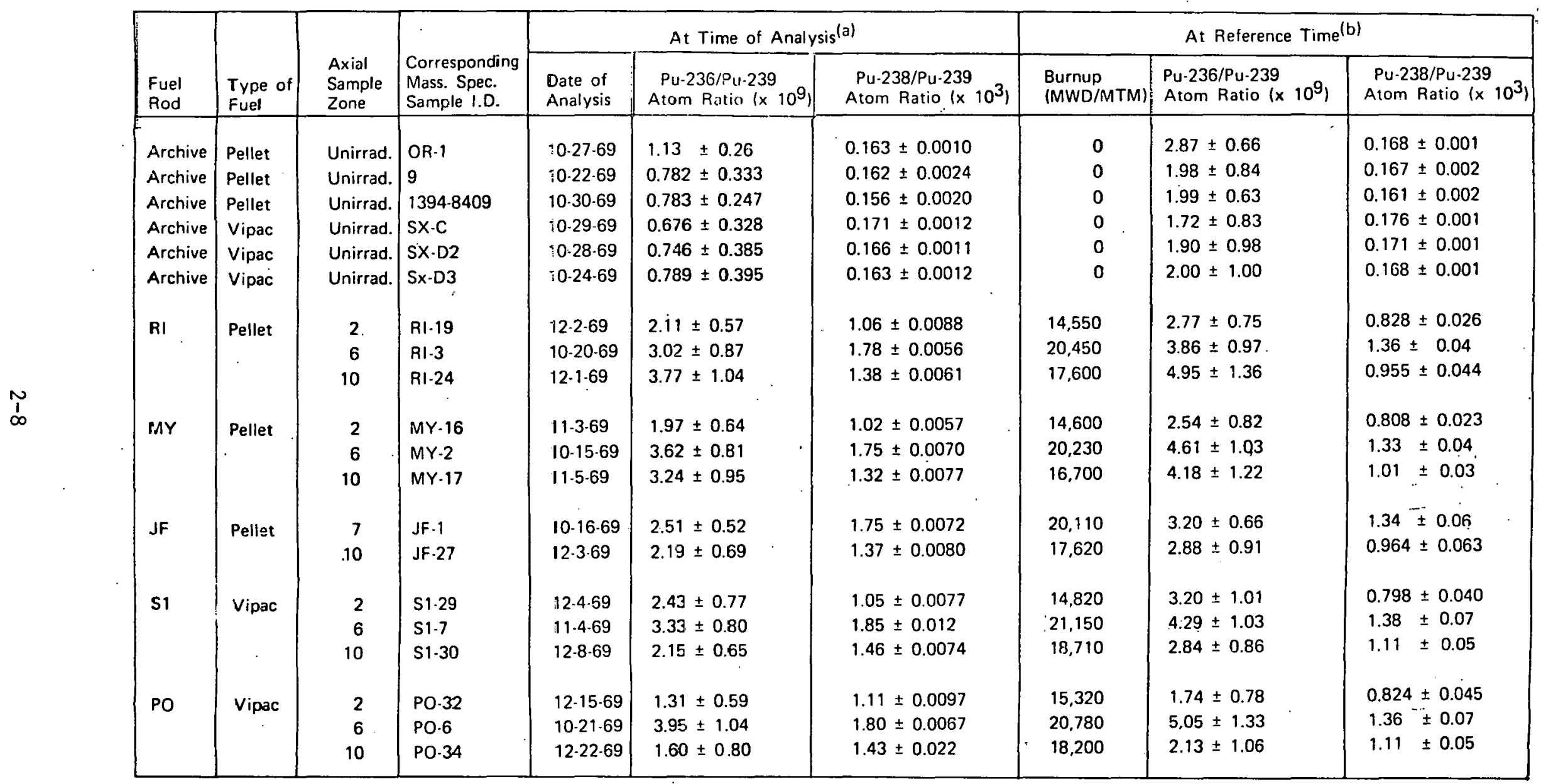

Notes: (a) Alpha spectrometry; errors shown are \pm two standard deviations, based on alpha counts.

(b) Results of alpha spectrometric analyses; unirradiated samples are referenced to Saxton Core II Beginning-of-life on December 28, 1965: irradiated samples are referenced to Core II end-of-life on October 18, 1968. 
TABLE 2-3

SUMMARY OF RADIOCHEMICAL ANALYSIS FOR Np-237 IN SAXTON CORE II FUEL

\begin{tabular}{|c|c|c|c|c|c|}
\hline $\begin{array}{l}\text { Fuel } \\
\text { Rod }\end{array}$ & $\begin{array}{l}\text { Axial } \\
\text { Sample } \\
\text { Zone }\end{array}$ & $\begin{array}{l}\text { Np-237(a) } \\
\text { Activity } \\
\text { dpm/gU }\left(\times 10^{-5}\right)\end{array}$ & $\begin{array}{l}\text { Corresponding } \\
\text { Mass Spec. } \\
\text { Sampie No. }\end{array}$ & $\begin{array}{l}\text { U-238 Weight } \\
\text { Fraction in } \\
\text { Total } U\end{array}$ & $\begin{array}{l}\text { Np-237iU-238 }(\mathrm{b}) \\
\text { Atom Ratio }\left(\times 10^{4}\right)\end{array}$ \\
\hline \multirow[t]{3}{*}{ RI } & 2 & 1.34 & $\mathrm{RI}-19$ & 0.99362 & 0.865 \\
\hline & 6 & 1.78 & RI-3. & 0.99398 & 1.15 \\
\hline & 10 & 1.16 & RI-24 & 0.99375 & 0.749 \\
\hline \multirow[t]{3}{*}{ MY } & 2 & 1.14 & MY-16 & 0.99361 & 0.736 \\
\hline & 6 & 1.76 & MY-2 & 0.99393 & $\uparrow .14$ \\
\hline & 10 & 1.59 & $M Y-17$ & 0.99375 & 1.03 \\
\hline
\end{tabular}

Notes: (a) Radiochemical analysis; all results $\pm 15 \%$, on November 26, 1969.

(b) $\quad N^{37} / N^{28}=A^{37} W^{28} / \lambda^{37} N_{a}(w f)^{28}$

where:

$$
\begin{aligned}
A^{37} & =\mathrm{Np}-237 \mathrm{dpm} / \mathrm{gU} \\
W^{28} & =\text { atomic weight U-238 } \\
\lambda^{37} & =\mathrm{Np}-237 \text { decay constant }\left(6.16 \times 10^{-13} \mathrm{~min}^{-1}\right) \\
\mathrm{N}_{\mathrm{a}} & =\text { Avogadro's Number } \\
(\mathrm{wf})^{28} & =\mathrm{U}-238 \text { weight fraction }
\end{aligned}
$$


TABLE 2-4

SUMMA.RY OF ANALYSES FOR AM-241. AND AM-243 IN SAXTON CORE II FUEL (e)

\begin{tabular}{|c|c|c|c|c|c|c|c|}
\hline \multirow[b]{2}{*}{$\begin{array}{l}\text { Fuel } \\
\text { Rod }\end{array}$} & \multirow{2}{*}{$\begin{array}{l}\text { Axial } \\
\text { Sample } \\
\text { Zone }\end{array}$} & \multicolumn{2}{|c|}{ Atom Ratio at Time of Arialysis $\left(\times 10^{2}\right)$} & \multicolumn{3}{|c|}{ Corresponding Mass Spectrometric Data } & \multirow{2}{*}{$\begin{array}{l}\text { Am-241/Pu-239(d) } \\
\text { Atom Ratio }\left(\times 10^{2}\right) \\
\text { at EOL }\end{array}$} \\
\hline & & $\frac{\text { Atom Ratio at Tin }}{\text { Am-241/Pu-239(a) }}$ & $\frac{\text { of Arialysis }\left(\times 10^{2}\right)}{A m-243 / \mathrm{Pu}-239^{(b)}}$ & $\begin{array}{l}\text { Sample } \\
\text { I.D. }\end{array}$ & $\begin{array}{l}\text { Date of } \\
\text { Analysis }\end{array}$ & $\begin{array}{l}\mathrm{Pu}-241 / \mathrm{Pu}-239(\mathrm{c}) \\
\text { Atom Ratio }\left(\times 10^{2}\right)\end{array}$ & \\
\hline \multirow[t]{3}{*}{$\mathrm{RI}$} & 2 & 0.845 & $<0.10$ & RI-19 & $11-10-69$ & 5.48 & $0.535 \pm 0.127$ \\
\hline & 6 & 1.27 & $<0.80$ & RI-3 & $9-3-69$ & 8.19 & $0.812 \pm 0.191$ \\
\hline & 10 & 1.35 & $<0.15$ & RI-24 & $11-17-69$ & 6.80 & $0.965 \pm 0.203$ \\
\hline \multirow[t]{3}{*}{ MY } & 2 & 0.840 & $<0.23$ & MY-16 & $10-21-69$ & 5.53 & $0.528 \pm 0.126$ \\
\hline & 6 & 1.23 & $<0.84$ & MY-2 & $9-20-69$ & 8.13 & $0.774 \pm 0.185$ \\
\hline & 10 & 1.04 & $<0.59$ & MY-17 & $11-4-69$ & 6.68 & $0.663 \pm 0.156$ \\
\hline
\end{tabular}

Notes: (a) Alpha spectrometric analysis on November 18, 1969; uncertainties are $\pm 15 \%$.

(b) - Alpha spectrometric analysis on December 18, 1969. The alpha spectrum resolution obtained for this set of samples was not sufficient to detect the Am-243 peak in the presence of the relatively large Am-241 peak; therefore, only "less than" values can be given.

(c) At time of mass spectrometric analysis; relative error is approximately \pm 0.3 percent.

(d) Referenced to Core II and-of-life on October 18, 1968; uncertainty is estimated two-standards deviation, and includes uncertainty in Pu-241 measurement.

(e) The atom ratio Am-241/Pu-239 in unirradiated fuel at Core II beginning-of-life on December 28, 1965 is estimated to be $8.34 \times 10^{-4}$, and is based on estimated value of Pu-241/Pu-239 atom ratio of $1.05 \times 10^{-2}$ at time of plutonium separation on or about May 15, 1964 
TABLE 2-5

SUMMARY OF ALPHA SPECTROMETRIC ANALYSIS FOR Cm-242/Pu-239 and $\mathrm{Cm}-244 / \mathrm{Pu}-239$ ATOM RATIOS IN SAXTON CORE II FUEL

\begin{tabular}{|c|c|c|c|c|c|c|c|c|}
\hline \multirow{2}{*}{$\begin{array}{l}\text { Fuel } \\
\text { Rod }\end{array}$} & \multirow{2}{*}{$\begin{array}{l}\text { Axial } \\
\text { Sample } \\
\text { Zone }\end{array}$} & Atom Ratio $\left(\times 10^{4}\right)$ at & Time of Analysis $(a)$ & \multirow{2}{*}{$\begin{array}{l}\text { Corresp. } \\
\text { Mass Spec. } \\
\text { Sample I.D. }\end{array}$} & \multirow{2}{*}{$\frac{N^{49^{(b)}}}{N^{28}}$} & \multirow{2}{*}{$\frac{N^{28^{(b)}}}{N^{28}}$} & \multicolumn{2}{|c|}{$\begin{array}{l}\text { Atom Ratio }\left(\times 10^{4}\right) \\
\text { At Core } 11 \mathrm{EOL}^{(\mathrm{c})}\end{array}$} \\
\hline & & \begin{tabular}{l|l}
$\mathrm{Cm}-242 / \mathrm{Pu}-239$ &
\end{tabular} & $\mathrm{Cm}-244 / \mathrm{Pu}-239$ & & & & $\mathrm{Cm}-242 / \mathrm{Pu}-239$ & $\mathrm{Cm}-244 / \mathrm{Pu}-239$ \\
\hline \multirow[t]{3}{*}{ RI } & 2 & 0.556 & 0.403 & $\mathrm{RI}-19$ & 0.04940 & 0.99262 & 2.88 & 0.420 \\
\hline & 6 & 1.06 & 1.24 & $\mathrm{RI}-3$ & 0.04410 & 0.98921 & 5.48 & 1.29 \\
\hline & 10 & 1.01 & 1.21 & $\mathrm{RI}-24$ & 0.04639 & 0.99091 & 5.23 & 1.26 \\
\hline \multirow[t]{3}{*}{ MY } & 2 & 0.522 & 0.366 & MY-16 & 0.04902 & 0.99292 & 2.70 & 0.381 \\
\hline & 6 & 1.05 & 1.09 & MY-2 & 0.04383 & 0.98986 & 5.43 & 1.14 \\
\hline & 10 & 0.757 & 0.712 & MY-17 & 0.04642 & 0.99127 & 3.92 & 0.742 \\
\hline
\end{tabular}

Notes: (a) Alpha spectrometric analysis on November 11, 1969. Relative uncertainties are $\pm 10 \%$ for $\mathrm{Cm}-242$ and $\pm 20 \%$ for $\mathrm{Cm}-244$.

(b) Pu-239 and U-238 atom ratios are shown here for reference.

(c) Referenced to Core 11 end-o:-life on October 18, 1968. 
TABLE 2-6

COMPARISON BETWEEN MEASUREMENT AND LEOPARD-HIC CALCULATION OF $\mathrm{Np}, \mathrm{Pu}, \mathrm{Am}$, AND $\mathrm{Cm}$ ISOTOPES IN SAXTON CORE II MIXED-OXIDE FUEL

\begin{tabular}{|c|c|c|c|}
\hline & Measured Parameter & $\begin{array}{l}\text { Percent }{ }^{\text {[a] }} \\
\text { Relative } \\
\text { Uncertainty } \\
\text { in Measurement }\end{array}$ & $\begin{array}{l}\text { Percent }{ }^{\text {Mb] }} \\
\text { Difference Between } \\
\text { Calculation and } \\
\text { Measurement }\end{array}$ \\
\hline Isotope & Np-237 dpm/g U & \pm 15 & -34 \\
Pu-236 & Pu-236/Pu-239 & \pm 24 & 8 \\
Pu-238 & Pu-238/Pu-239 & \pm 4 & -28 \\
Am-241 & Am-241/Pu-239 & \pm 24 & -4 \\
Am-243 & Am-243/Pu-239 & {$[\mathrm{c}]$} & -- \\
Cm-242 & Cm-242/Pu-239 & \pm 10 & -24 \\
Cm-244 & Cm-244/Pu-239 & \pm 20 & -38 \\
\hline
\end{tabular}

Notes: (a) Two standard deviations. Referenced to core end-of-life.

(b) (Calc-Meas)/Meas $\times 100$ percent; LEOPARD-HIC axial Zone 6 calculation at about 20,000 MWD/MTM.

(c) Measured data not resolved. 


\section{SECTION 3}

CORE III OPERATIONS

(C. E. Palmer, R. L. Stover)

The reactor was shut down from the beginning of the period until. August 24 , 1970 for the examination of fuel rods from removable assemblies. It was started up on August 24 and operated at 20.8 MWt until August 28 . The power was increased to 22.5 MWt on August 28 and continued at this level until September 7, when the reactor was shut down for repair of a flux thimble drive. Following the repair, the reactor was returned to power on September 10 and operated at 23.6 MWt (100\% power) until September 18, when it was shut down, as planned, for operator training.

As of the last shutdown, on September 18, the cumulative energy generated in Core III was 2133 megawatt-days, with $448 \mathrm{MWD}$ since August 24. During the period from August 24 to September 18, forty-six additional load follow cycles were achieved, bringing the total to 184 since the beginning of Core III. A series of flux maps were obtained to determine if radial or axial power transients occurred during load cycling between 100 percent and 40 percent of peak power. With control rod number 2 moving between 17 inches and 14 inches (fully out position is 36.6 inches), radial variations were \pm 3 percent and axial variations only \pm 1 percent. As a result, it is concluded that no significant power transients occurred during this mode of load cycling.

Flux map data were also analyzed to provide relative assembly and rod power levels during power operation in mid-September at $2800 \mathrm{MWD} / \mathrm{MTM}$ average core burnup. Figure 3-1 shows the measured values for all assemblies in the core and compares them with the predicted power levels. Table 3-1 summarizes the operating history of key rods in the loose lattice region of Core III during the past quarter; it includes the best estimate peak linear power and burnup of the lead rods. 
(1) PREDICTED

(2) MEASURED

(3) $\frac{\text { MEASURED - PREDICTED }}{\text { MEASURED }} \times 100$

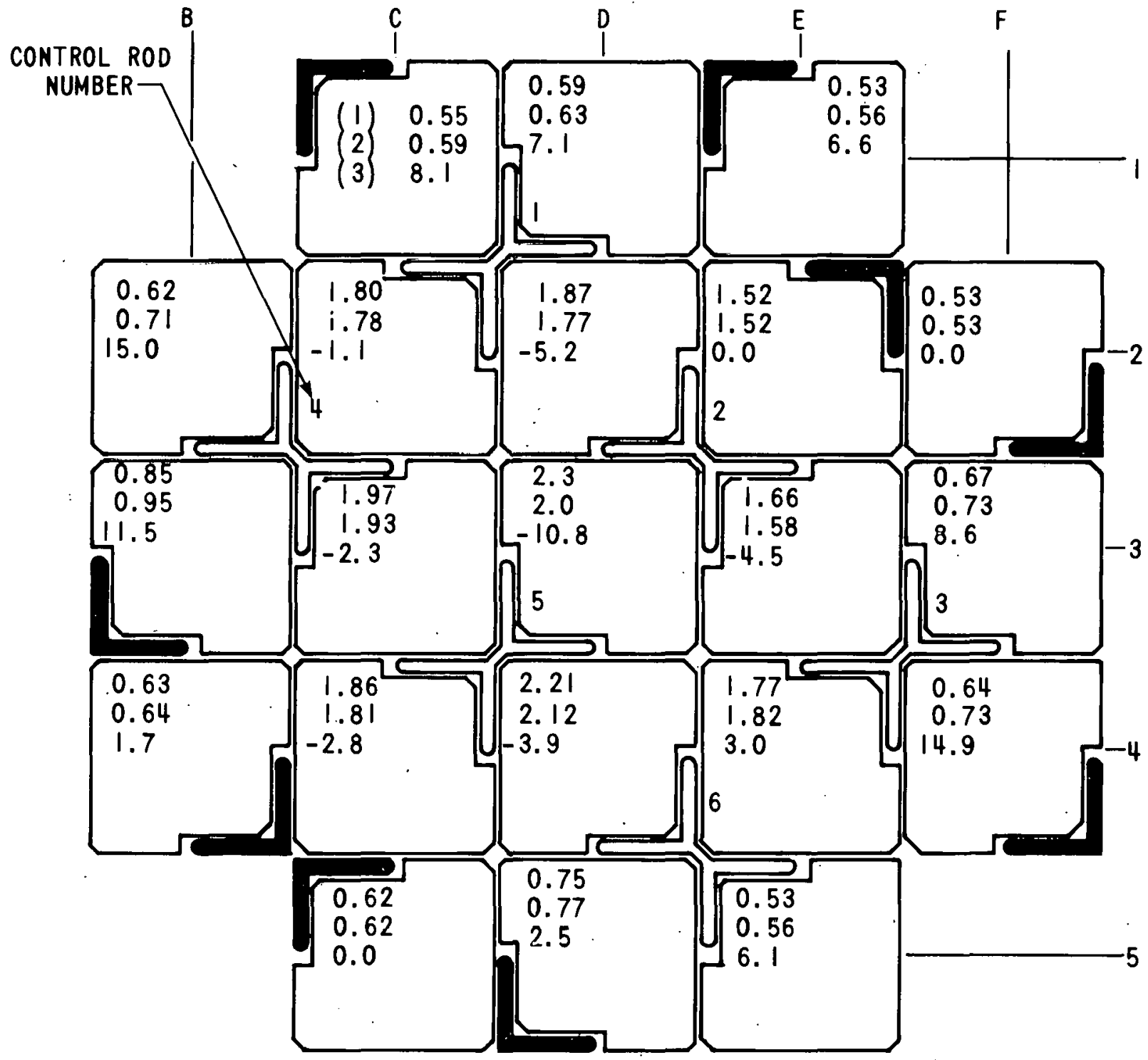

DATA FROM FLUX MAP NO: 310.3.35 (SEPT. 14, 1970)

ROD $2=17$ INCHES (OUT = 36.6 INCHES)

AVERAGE CORE BURNUP $=2800 \mathrm{MWD} / \mathrm{MTU}$

Figure 3-1. Relative Assembly Power for Saxton Core III 
TABLE $3-1$

SUMMARY OF SAXTON CORE III OPERATING HISTORY - THIRD QUARTER 1970

\begin{tabular}{|c|c|c|c|}
\hline 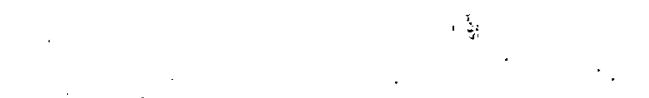 & $\begin{array}{l}\text { Through } \\
\text { June } 30\end{array}$ & $\begin{array}{l}\text { During } \\
\text { Aug. \& Sept. }\end{array}$ & $\begin{array}{l}\text { Cumulative } \\
\text { to Sept. } 30 .\end{array}$ \\
\hline Energy Generated, MWD & 1,685 & 448 & 2,133 \\
\hline Number of Load Cycles & 138 & 46 & 184 \\
\hline $\begin{array}{l}\text { Peak Linear Power, kw/ft }(\mathrm{a}) \\
\text { 1. Peak Power Rod } \\
\text { 2. Peak Burnup Rod } \\
\text { (in center } 3 \times 3 \text { ) } \\
\text { 3. Peak Burnup Rod } \\
\text { Outside Center } 3 \times 3\end{array}$ & $\begin{array}{l}19.0^{(\mathrm{c})} \\
15.6^{(\mathrm{c})} \\
9.8^{(1}\end{array}$ & $\begin{array}{c}18.8 \\
15.3 \\
9.6\end{array}$ & $\begin{array}{c}-\bar{c} \\
-\infty \\
-\infty\end{array}$ \\
\hline $\begin{array}{l}\text { Peak Pellet Burnup, MWD/MTM(b) } \\
\text { 1. Peak Power Rod } \\
\text { 2. Peak Burnup Rod } \\
\text { 3. Peak Burnup Rod } \\
\text { Outside Center } 3 \times 3\end{array}$ & $\begin{array}{l}25,300 \\
35,200^{(c)}\end{array}$ & $\begin{array}{l}2,300 \\
1,900\end{array}$ & 37,100 \\
\hline
\end{tabular}

(a) Best estimate, thermal basis (thermal $=0.974$ of fission)

(b) Best estimate, fission basis

(c) Corrected values based on re-analysis of flux data, using up-dated constants 


\section{SECTION 4}

\section{EVALUATION OF CORE III FUEL}

(J. T. Barnes, E. S. Schwartz, W. R. Smalley, R. S. Miller)

\subsection{EVALUATION OF ROD RR}

Rod RR was removed from the center $3 \times 3$ subassembly during the interim shutdown and transferred to the WPIF Hot Laboratory. During the period, the rod was visually examined in detail and gamma scanned.

Visual examination showed that the overall appearance of rod RR was similar to that of the highest power rods removed at the end of Core II. Prior to cleaning, the rod exhibited uniformly light to moderately thick crud over most of its surface. It also showed the usual scratches and score marks, which resulted from removal of the rod from the subassembly and subsequent handling operations. After cleaning, the major surface features were patchy areas of non-uniform color over most of the center 24 inches of the rod. These areas were characterized by alternately dark and light zones, both of irregular shape. Generally, the dark areas were non-lustrous while the lighter ones were somewhat shiny. These conditions are illustrated in Figure 4-1. Another feature was the transition from the patchy condition to a uniform, semi-lustrous black surface in the areas of the second and third grids, extending approximately 2 inches in each case. A typical area illustrating such a transition is shown in Figure 4-2. The top and bottom 7 inches of the rod appeared to be generally black and uniformly lustrous, except for grid contact marks and handling scratches. The visual examination of rod RR. revealed no evidence of swelling, hydride blisters, cracks, or other defects. After visual examination, the rod was scanned with a sodium iodide scintillation crystal detector to determine gross gamma activity at quarter-inch intervals. The resulting data are being evaluated to help identify the location of samples for subsequent metallographic examination and burnup analysis. 


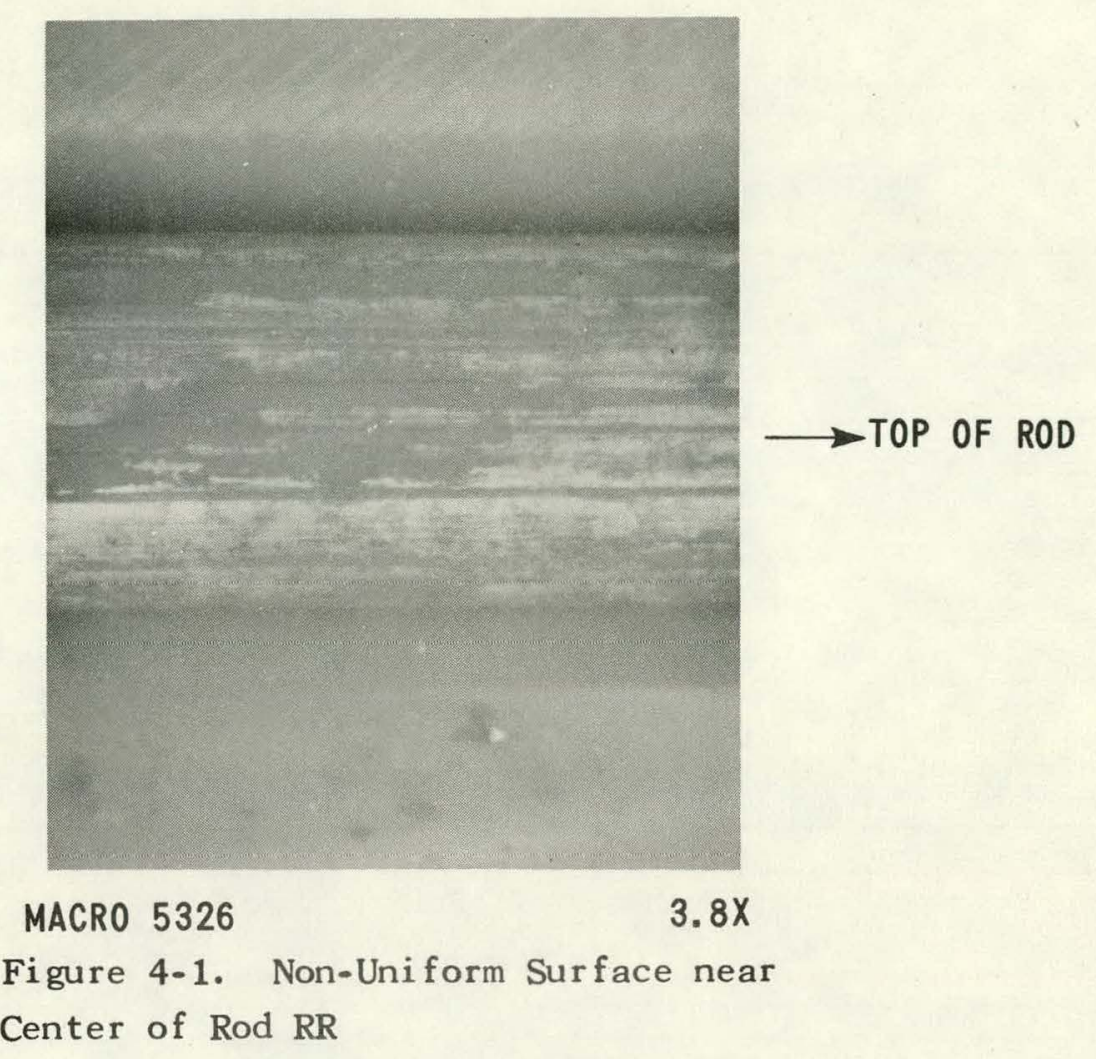

\section{$3.8 \mathrm{X}$}

Figure 4-2. Transition Area near Grid, 23.8 Inches from Bottom of Rod RR 


\subsection{MID-LIFE SAMPLING PLAN}

At the mid-1ife shutdown of Core III, seven fuel rods will be removed from two loose lattice assemblies and sent to the WPIF Hot Laboratory for destructive examination. The seven rods selected are listed in Table 4-1.

TABLE 4-1

LOOSE LATTICE FUEL RODS TO BE REMOVED AT MID-LIFE FOR DESTRUCTIVE EXAMINATION

\begin{tabular}{|c|c|c|c|}
\hline $\begin{array}{c}\text { Assembly } \\
\text { Serial Number }\end{array}$ & $\begin{array}{c}\text { Core III } \\
\text { Lattice Position } \\
\text { Assembly-Rod }\end{array}$ & $\begin{array}{c}\text { Serial } \\
\text { Number* }\end{array}$ & $\begin{array}{c}\text { Best Estimate Peak } \\
\text { Linear Power, kw/ft }\end{array}$ \\
\hline $503-17-2$ & D2-J1 & BO & $19-20$ \\
$503-17-2$ & D2- J3 & NE & $19-20$ \\
$503-17-2$ & D2- H2 & NI & $17-18$ \\
$503-17-2$ & D2-D8 & GL & $14-15$ \\
$503-17-9$ & E4- D5 & FS & $14-15$ \\
$503-17-9$ & E4- G2 & MQ & $9-10$ \\
$503-17-9$ & E4- J2 & FP & $9-10$ \\
\hline
\end{tabular}

*Identification stamped on horizontal surface of top end plug. 